\title{
Estimation of Bone-on-Bone Forces at the Tibiofemoral Joint during Forward and Backward Stair Descent
}

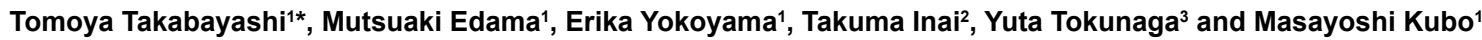 \\ ${ }^{1}$ Institute for Human Movement and Medical Sciences, Niigata University of Health and Welfare, 1398 Shimami-cho, Kita-Ku, Niigata City, Niigata, 950-3198, Japan \\ ${ }^{2}$ Oguma Orthopedics Clinic, Niigata, Japan
}

${ }^{3}$ Sekikawa Aikoen, Geriatric Health Services Facilities, Niigata, Japan

\begin{abstract}
Objective: Backward stair descent has attracted attention from the viewpoint of injury prevention. Previous studies have shown that knee extension moment is lower during the backward stair descent than during the forward stair descent. However, it remains unclear whether the bone-on-bone force at the tibiofemoral joint differs between the two stair descent methods. The purpose of this study was to estimate bone-on-bone forces at the tibiofemoral joint during forward and backward stair descent.
\end{abstract}

Methods: Six healthy young men participated in this study. The stair descents were performed in the forward and backward direction using a step-by-step pattern. The muscle tensions of the lower limbs were estimated based on the net joint moments and information provided by the electromyography. The bone-on-bone forces at the tibiofemoral joint were calculated based on the estimated muscle tensions and geometry of limbs.

Results: While the knee extension moments were significantly lower during backward stair descent than forward stair descent, the estimated bone-on-bone forces at the tibiofemoral joint in two different descending methods showed no significant difference. The quadriceps femoris tension during backward stair descent was significantly lower than that during forward stair descent.

Conclusions: The bone-on-bone forces reflect mechanical load to the articular surface. Thus, the results of this study suggested that the load of tibiofemoral articular surface remains unchanged between the forward and backward stair descent. However, backward stair descent might be a relevant method for reduction of quadriceps femoris tensions.

Keywords: Bone-on-bone force; Tibiofemoral joint; Stair descent; Net joint moment

\section{Introduction}

Knee Osteoarthritis (OA) is a common, disabling, and expensive disease which predominantly effects on the elderly [1] and has significant impact on the activities of daily living and the quality of life of the patients. Knee OA weakens tissues of the joint with age, including the articular cartilage, joint meniscus, and ligaments. Repeated overloading of the tibiofemoral joint is a factor that contributes to the deterioration of the articular cartilage and associated with initiation and progression of knee OA. Because self-healing is difficult once the articular cartilage has deteriorated [2], patients with knee OA need to minimize tibiofemoral joint overloading in daily activities.

One of the most difficult activities of daily life for patients with knee OA is stair descent [3]. Stair descent requires higher knee moment with more eccentric contraction compared to stair ascent [4,5]. In order to decrease knee joint loads, patients with knee OA are often instructed to use a step-by-step pattern (placing both feet on the same step before descending) during forward stair descent (FD) by putting the more affected limb down first. As an alternative strategy to the FD, backward stair descent (BD) has been suggested as a method of injury prevention. For example, a previous study [6] demonstrated that knee extension moment is lower during $\mathrm{BD}$ than during $\mathrm{FD}$. Thus, the $\mathrm{BD}$ may offer a less stressful and safer means of descending stairs under certain circumstance.

Furthermore, it has been reported that the different step patterns afferent kinematics of the knee joint. Reid et al. showed stair descent with a step-by-step pattern lowered knee moment and knee power in sagittal plane compared to that with a step-over-step pattern. Thus, a step-by-step pattern may be suitable for clinical relevance.
One of the indicators of the loads to joint is the joint moments derived by a method of the inverse dynamics. This kinetic variable was often used in various studies of movement such as gait [7], squat [8], running [9] as well as stair descent [6]. However, the joint moments are a net effect of tensions generated by antagonist and agonist muscles and may not reflect the amount of mechanical load generated at the articular surface. For instance, when antagonist and agonist muscles produce the same amount of moments, the net joint moment would be zero (e.g. co-contraction).

Bone-on-bone forces are used as an indicator of the mechanical load on the articular surface [7]. The estimation of these forces requires the measurement of muscle tensions crossing the joint and joint reaction forces. While joint reaction forces can be derived from a method of inverse dynamics, individual muscle tension is difficult to measure. Electromyography (EMG) assisted optimization (EAO) is one of noninvasive methods for estimating muscle tensions by

*Corresponding author: Tomoya Takabayashi, Institute for Human Movement and Medical Sciences, Niigata University of Health and Welfare, 1398 Shimamicho, Kita-Ku, Niigata City, Niigata, 950-3198, Japan, Tel: +81-25-257-4455; Fax +81-25-257-4456; E-mail: hwd14005@nuhw.ac.jp

Received November 19, 2015; Accepted December 10, 2015; Published December 17,2015

Citation: Takabayashi T, Edama M, Yokoyama E, Inai T, Tokunaga Y, et al. (2015) Estimation of Bone-on-Bone Forces at the Tibiofemoral Joint during Forward and Backward Stair Descent. J Arthritis 4: 181. doi:10.4172/2167-7921.1000181

Copyright: (c) 2015 Takabayashi T, et al. This is an open-access article distributed under the terms of the Creative Commons Attribution License, which permits unrestricted use, distribution, and reproduction in any medium, provided the original author and source are credited. 
combining the mathematical optimization of the joint torques and the information provided by surface EMG. It has been reported that the EAO approach can estimate muscle tensions even under concurrent contractions $[10,11]$.

Estimation of muscle tensions by the EAO approach with information on the limb geometry in combination with the joint moment's inverse dynamics allows us to evaluate the bone-on-bone joint forces. The purpose of this study was to estimate the bone-onbone force at the tibiofemoral joint of the leading leg during both FD and BD using a step-by-step pattern.

\section{Methods}

\section{Subjects}

Six healthy male subjects with similar body height $(172.1 \pm 2.8$ $\mathrm{cm})$, weight $(65.7 \pm 5.6 \mathrm{~kg})$ and age $(23.8 \pm 2.6$ years $)$ participated in this study. They were free of any musculoskeletal or neurological dysfunctions that may affect the performance of the stair descent. The Niigata University of Health and Welfare (No. 17324-120605) internal review board approved this study. All subjects gave their written informed consent prior to participation.

\section{Experimental procedure}

The subjects descended the stairs placed on the force platform with bare feet at comfortable speed. The stair descents were performed in forward and backward using the step-by-step pattern involving dual footfalls at each step (Figure 1). The focus of the analysis was on the leading leg (right leg in this study). The gait cycle in this study was defined by two consecutive toe contacts of the foot of the leading leg. Prior to data acquisition, the participants were allowed to practice the stair descent repeatedly until they were accustomed to two different methods. A participant performed both the forward and backward stair descents in random order until 5 trials were obtained for each condition. The stairs used in this study consisted of 5 steps and complied with the barrier-free law of Japan (i.e., riser: $160 \mathrm{~mm}$, tread: $300 \mathrm{~mm}$ ).

Descending motions were captured by a 3D motion analysis system (Vicon, Oxford, UK) that included 11 infrared cameras and 6 force platforms (AMTI Inc, Japan) with a sampling rate of $100 \mathrm{~Hz}$ and $1 \mathrm{KHz}$, respectively. Surface EMG was obtained from 7 muscles of the leading leg at $1 \mathrm{KHz}$ sampling rate, including the rectus femoris (RF), vastus
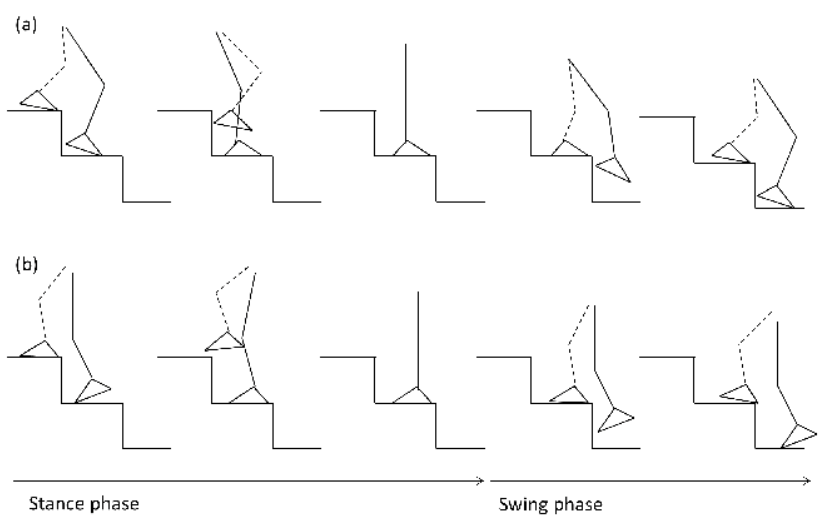

Figure 1: Methods of forward (a) and backward stair descent (b) with stepby-step pattern. The focus of the analysis was on the leading leg (solid line). medialis (VM), long head of biceps femoris (BFL), semitendinosus (ST), tibialis anterior (TA), gastrocnemius (GAS), and soleus (SOL).

Joint angles, net joint moments, and joint reaction forces of the knee and ankle joints were calculated using the Bodybuilder (OMG PLC, UK) followed by zero-lag Butterworth low-pass filter with a 6 $\mathrm{Hz}$ cut off frequency. Surface EMG data was processed with zero-lag Butterworth high-pass filter with a $20 \mathrm{~Hz}$ cut off, and then full wave rectified. The linear envelope of the surface EMG was created by applying a low-pass filter with a $6 \mathrm{~Hz}$ cut-off and then down sampled at $100 \mathrm{~Hz}$ to ensure synchronization with other kinetic and kinematic data. Processed surface EMG data was normalized to that of the isometric maximum voluntary contraction of each muscle. All trial data were time normalized to the gait cycle.

\section{Estimation of muscle tensions}

The muscle tensions were estimated by the modified version of EAO $[10,11]$ which involved 9 muscles including the RF, Vastus (VAS), ST, semimembranosus muscle (SM), BFL, short head of the biceps femoris (BFS), as well as the TA, GAS and SOL.

The tentative tension of $i$ th muscle $F_{i}^{\prime}$ was a product of the muscle's physiological cross section areas $\left(P C S A_{i}\right)$ maximal muscle stress $(\sigma$ : $\left.50 \mathrm{~N} / \mathrm{m}^{2}\right)[12]$ and the normalized surface EMG activity of the muscles $\left(\% M V C_{i}\right)$ (Equation 1). In this study, the values of PCSA $A_{i}$ were obtained from the previous study [13].

$$
F_{i}^{\prime}=P C S A_{i} \sigma \% M V C_{i},(i=1 \ldots 9)
$$

The tentative moment of $j$ th joint generated by $i$ th muscle $\left(M_{j}\right)$ was estimated as a cross product of the lever arm vector $\left(L_{j i}\right)$ [14-17] and the $F_{i}^{\prime}$ (Equation 2). There were two joints involved in the equation (knee and ankle).

$$
M_{j i}^{\prime}=L_{j i} \times F_{i}^{\prime},(j=1 \ldots 2)
$$

Tentative moments were adjusted by the gain $\left(g_{i}\right)$ so that the sum of the adjusted moments became equivalent to the joint moment $\left(M_{j}\right)$ obtained by the inverse dynamics method (Equation 3 ).

$$
\mathrm{M}_{\mathrm{j}}=\mathrm{g}_{\mathrm{i}} \mathrm{M}_{\mathrm{j}} \mathrm{i}^{\wedge \prime},\left(g_{i} \geq 0\right)
$$

The set of gains $\left(g_{i}\right)$ were also needed in order to minimize the objective function $(I)$ shown in equation 4 .

$$
J=\sum_{\mathrm{j}=1}^{2} \sum_{\mathrm{i}=1}^{9}\left|M_{j i}^{\prime}\right|\left(1-g_{i}\right)^{2}
$$

The individual muscle tension $\left(F_{i}\right)$ was determined as a product of gain $\left(g_{i}\right)$ and tentative muscle tension $\left(F_{i}\right)$ (Equation 5).

$$
F_{i}=g_{i} F_{i}^{\prime}
$$

Estimation of bone on bone forces at the tibiofemoral joint

Extensor tension $\left(F_{\mathrm{EXT} / /}\right)$ was calculated as the sum of the RF and VAS tensions. Flexion tension $\left(F_{\mathrm{EXT} / /}\right)$ was calculated as the sum of the medial (HAMM: ST and SM) and lateral hamstrings tensions (HAML: BFL and BFS) and GAS. TA and SOL tensions were excluded because they are not associated with the tibiofemoral joint. The force components of these summed forces parallel to the long axis of the tibia were expressed as $\left(F_{\mathrm{EXT} / /}\right)$ and $\left(F_{\mathrm{FLE} /}\right)$, respectively.

The bone-on-bone forces at the tibiofemoral joint $\left(F_{T F / /}\right)$ were calculated by a component of the tibiofemoral joint reaction force parallel to the long axis of the tibia $\left(F_{\mathrm{R} / /}\right)$ and the individual muscle tensions (Equation 6).

$$
F_{T F / /}=F_{R / /}-\left(F_{E X T / /}+F_{F L E / /}\right)
$$


Citation: Takabayashi T, Edama M, Yokoyama E, Inai T, Tokunaga Y, et al. (2015) Estimation of Bone-on-Bone Forces at the Tibiofemoral Joint during Forward and Backward Stair Descent. J Arthritis 4: 181. doi:10.4172/2167-7921.1000181

Page 3 of 5

$F_{T F / /}$ was normalized to the body mass. All the computation of this study was performed by using the Scilab-5.4.1 software (Inria, FR).

\section{Statistical analysis}

The differences between mean values of the maximal bone-on-bone forces, muscle tensions and the net joint moments at the tibiofemoral joint between the two descending conditions were evaluated by the Wilcoxon signed rank test. The statistical significance level was set at $5 \%(p<0.05)$.

\section{Results}

Figure 2 showed the bone-on-bone forces at the tibiofemoral joint over one gait cycle in the FD and $\mathrm{BD}$. The maximal bone-on-bone forces at the tibiofemoral joint were not significantly different between the FD and BD $(41.3 \pm 9.7 \mathrm{~N} / \mathrm{kg}$ at $13 \%$ of gait cycle and $44.8 \pm 11.4 \mathrm{~N} /$ $\mathrm{kg}$ at $17 \%$ of gait cycle, respectively). Overall, the bone-on-bone forces showed similar waveform patterns in two conditions. However, the maximal knee extension moment during $\mathrm{BD}(0.1 \pm 0.1 \mathrm{Nm} / \mathrm{kg}$ at $34 \%$ of the gait cycle) was significantly lower than that during FD $(0.3 \pm 0.2$ $\mathrm{Nm} / \mathrm{kg}$ at $16 \%$ of the gait cycle; Figure 3 ).

The estimated maximal RF and VAS tensions showed significantly lower values during BD than FD (Table 1). RF (Figure 4a), VAS (Figure $4 \mathrm{~b}$ ), and GAS (Figure 4c) tensions showed the maximal value from the

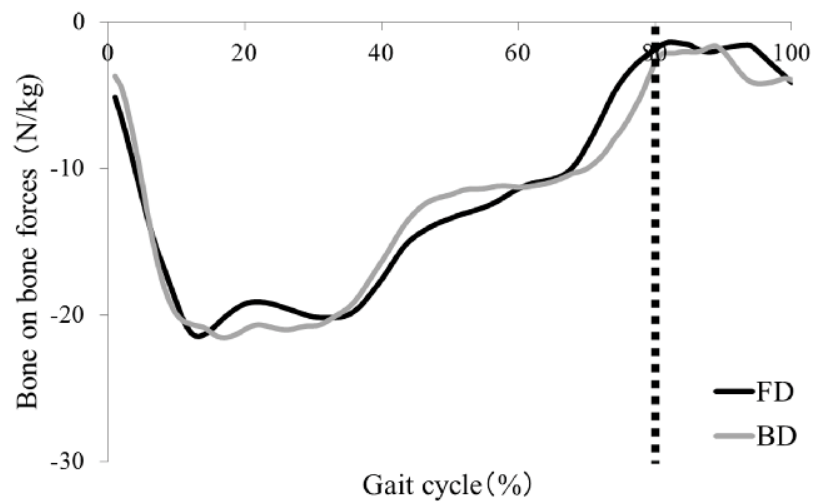

Figure 2: Bone-on-bone forces at the tibiofemoral joint during forward and backward stair descent. The vertical dashed line shows start of the swing phase. FD: Forward stair descent, BD: Backward stair descent.

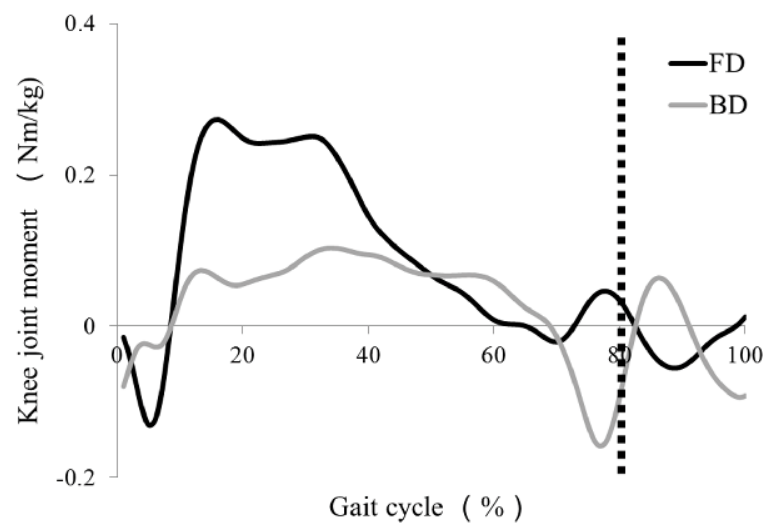

Figure 3: Knee joint moments during forward and backward stair descent. The vertical dashed line shows the start of the swing phase. Positive moment at the knee joint is extensor. FD; Forward stair descent, BD; Backward stair descent.

\begin{tabular}{|c|c|c|c|c|c|}
\hline \multicolumn{7}{|c|}{ Muscle tensions (N/kg) } \\
\hline Decending Method & RF* $^{*}$ & VAS $^{*}$ & HAMM & HAML $^{*}$ & GAS \\
\hline Forward & $4.9(3.0)$ & $5.7(1.8)$ & $2.2(0.7)$ & $2.0(0.8)$ & $4.4(1.2)$ \\
\hline Backward & $1.9(1.2)$ & $4.1(1.4)$ & $2.9(0.7)$ & $3.1(1.1)$ & $4.7(1.5)$ \\
\hline \multicolumn{7}{|c}{${ }^{*} \mathrm{p}<0.05$} \\
\hline
\end{tabular}

Table 1: Estimated maximal muscle tensions during the forward and backward stair descent. RF; Rectus femoris, VAS; Vastus, HAML; Lateral hamstrings, HAMM; Medial hamstrings, GAS; Gastrocnemius

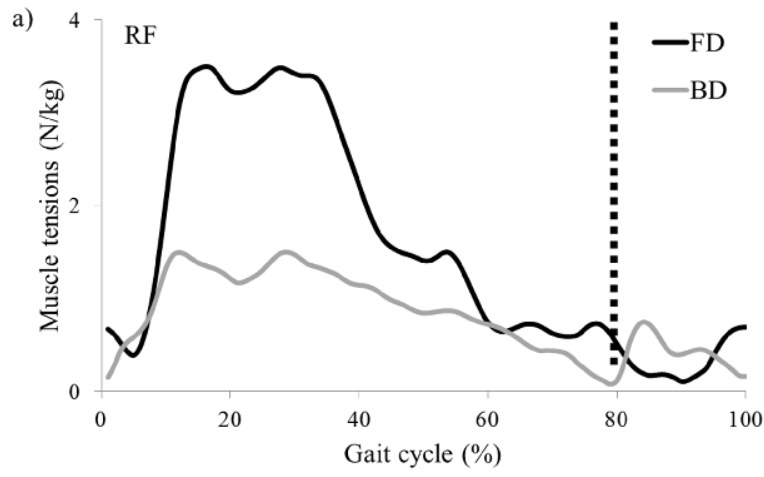

Figure 4a: Muscle tensions during the forward and backward stair descent. The vertical bar shows the start of the swing phase. FD; Forward stair descent, BD; Backward stair descent, a) RF; Rectus femoris.

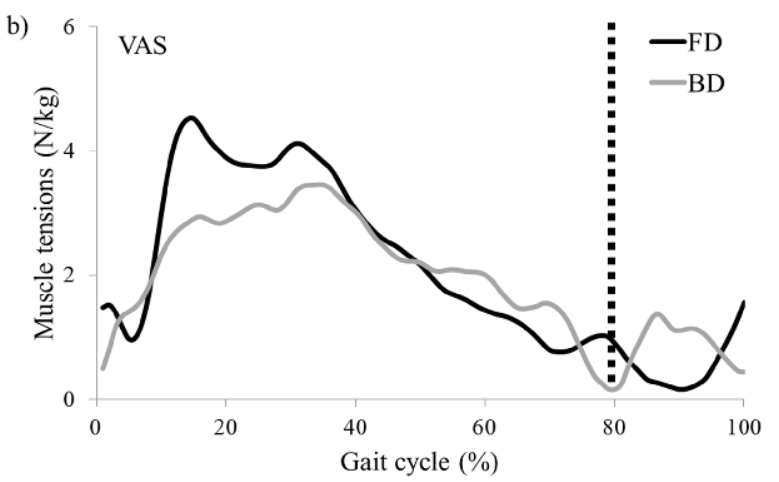

b) VAS; Vastus.

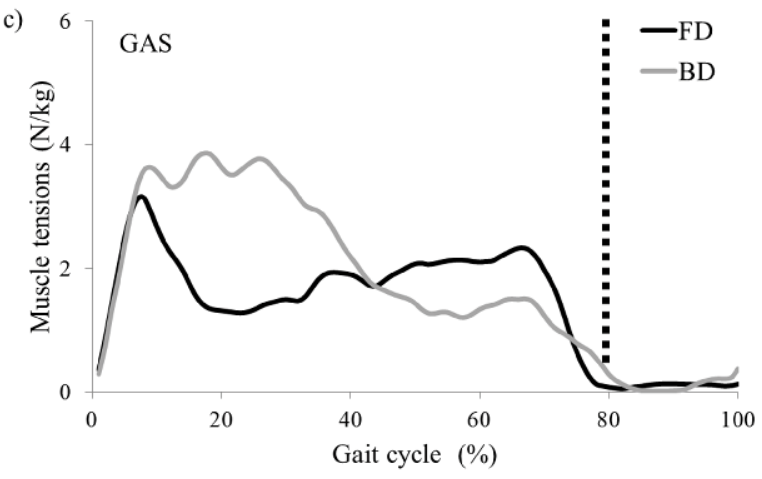

c) GAS; Gastrocnemius. 


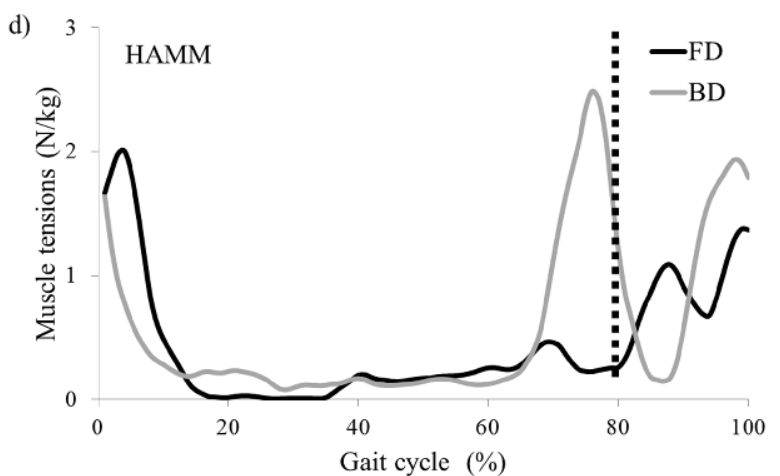

d) HAMM; Medial hamstrings

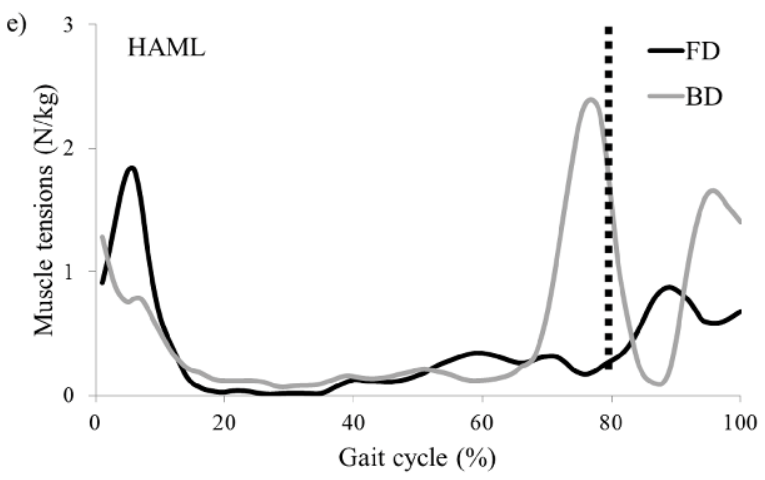

e) HAML; Lateral hamstrings.

toe contact to foot flat during both FD and BD. In contrast, HAMM (Figure 4d) and HAML (Figure 4e) showed maximal tensions at each level of the gait cycle. While they showed maximal values during toe contact to foot flat in the FD (at $4 \%$ and $6 \%$ of the gait cycle, respectively), the maximal values in $\mathrm{BD}$ were observed during preswing (at $76 \%$ and $77 \%$ of the gait cycle, respectively).

\section{Discussion}

The present study is the first to estimate bone-on-bone forces at the tibiofemoral joint during forward and backward stair descent. In spite of the significant differences in knee extension moments between FD and $\mathrm{BD}$ performed in a step-by-step manner, there were no significant differences in peak bone-on-bone forces at the tibiofemoral articular surface (at $5-20 \%$ of gait cycle). In present results, FD showed higher tensions in RF and VA compared to BD (at $5-20 \%$ of gait cycle). In contrast, BD showed higher tension in GAS (action of knee flexion) compared to FD (at $5-20 \%$ of gait cycle). While calculation of joint moments are net effect of tension generated by antagonist and agonist muscles, calculation of bone-on-bone forces are the sum of tensions produced by antagonist and aganist muscles [7]. In order to provide safe and efficient guidance to patients with knee OA, we need to understand the mechanical joint loads while performing stair descent. In view of present results, the bone-on-bone forces might be an appropriate choice.

While the bone-on-bone force does not change during $\mathrm{FD}$ and $\mathrm{BD}$, present study suggested that BD could decrease quadriceps femoris tension. In $\mathrm{FD}$, the estimated $\mathrm{RF}$ and VAS tensions were higher than those during BD. A previous study [18] has reported significant amounts of external knee flexion moments throughout the stance phase with peak values at initial contact and foot flat during FD. In order to counteract the external flexion moments due to gravity, the knee joint needs to generate high extension moments that require higher quadricep tensions. Clinically, since occurrence of hydrarthroses [19] and increased intramuscular fat [20] due to knee OA cause weakness of quadriceps femoris, the BD might be a more suitable method of descent for people with weak quadriceps femoris such as patients with knee OA and the elderly.

Furthermore, the results of present study may have a clinical implication for knee OA patients with patellofemoral pain. Patients with knee OA reported pain at the patellofemoral joint as well as at the tibiofemoral joint [21]. Since the quadriceps femoris tension contributes to the compressive force at the patellofemoral joint [22], the reduced tension of the knee extensors in BD might lead to the alleviation of pain and the prevention of articular cartilage lesions at the patellofemoral joint.

In this study, the estimation of bone-on-bone forces was limited to the sagittal plane. In patients with knee OA, the problem appears not only sagittal plane but also frontal planes, including knee varus deformity [2] and an increase in the external adduction moment during gait $[5,23]$. Therefore, the sagittal plane model would need to be expanded into a three-dimensional model (e.g. load of medial compartment) in the future. In addition, present study did not estimate shear forces at the tibiofemoral joint. Previous study [24] has reported the antero-posterior laxity of the knee $\mathrm{OA}$ in the early stage was greater than control group in knee extension task. Thus, not only compression forces but also shear forces may lead to deterioration of articular cartilage. However, same study [24] has suggested antero-posterior laxity decreases as OA stage progresses. Also, since movement of closed kinetic chain involves co-contraction of antagonist and agonist muscles, impact of the shear forces is less than that of compression. Lastly, since the present study involved only healthy young subjects, this result would not necessarily be generalizable to older patients with knee OA. We plan to investigate the difference the bone-on-bone forces at the tibiofemoral joint during forward and backward stair descent for patients with knee OA.

\section{Conclusion}

This study estimated bone-on-bone forces at the tibiofemoral joint of the leading leg using step-by-step during forward and backward stair descent. While knee extension moments were significantly lower during $\mathrm{BD}$ than $\mathrm{FD}$, the estimated bone-on-bone forces at the tibiofemoral joint showed no significant differences between the two stair descent methods. However, the quadriceps femoris tensions were significantly lower during BD than FD. These findings may be applied to clinical settings.

\section{Acknowledgements}

This study was supported by a Grant in Aid from the President of Niigata University of Health and Welfare Japan, 2013

\section{References}

1. van Saase JL, van Romunde LK, Cats A, Vandenbroucke JP, Valkenburg HA (1989) Epidemiology of osteoarthritis: Zoetermeer survey. Comparison of radiological osteoarthritis in a Dutch population with that in 10 other populations. Annals of the rheumatic diseases 48: 271-280.

2. Roddy E, Zhang W, Doherty M, Arden NK, Barlow J, et al. (2005) Evidencebased recommendations for the role of exercise in the management of osteoarthritis of the hip or knee--the MOVE consensus. Rheumatology (Oxford) 44: $67-73$. 
Citation: Takabayashi T, Edama M, Yokoyama E, Inai T, Tokunaga Y, et al. (2015) Estimation of Bone-on-Bone Forces at the Tibiofemoral Joint during Forward and Backward Stair Descent. J Arthritis 4: 181. doi:10.4172/2167-7921.1000181

Page 5 of 5

3. Hinman RS, Bennell KL, Metcalf BR, Crossley KM (2002) Delayed onset of quadriceps activity and altered knee joint kinematics during stair stepping in individuals with knee osteoarthritis. Archives of physical medicine and rehabilitation 83: 1080-1086.

4. Riener R, Rabuffetti M, Frigo C (2002) Stair ascent and descent at different inclinations. Gait Posture 15: 32-44.

5. Kaufman KR, Hughes C, Morrey BF, Morrey M, An KN (2001) Gait characteristics of patients with knee osteoarthritis. J Biomech 34: 907-915.

6. D Beaulieu FG, Pelland L, Robertson DG (2008) Kinetic analysis of forwards and backwards stair descent. Gait Posture 27: 564-571.

7. Winter DA (2009) Biomechanics and motor control of human movement (4th edtn) N.J Wiley, Hoboken, USA.

8. Almosnino S, Kingston D, Graham RB (2013) Three-dimensional knee joint moments during performance of the bodyweight squat: effects of stance width and foot rotation. J Appl Biomech 29: 33-43.

9. Belli A, Kyröläinen H, Komi PV (2002) Moment and power of lower limb joints in running. Int J Sports Med 23: 136-141.

10. Cholewicki J, McGill SM (1994) EMG assisted optimization: a hybrid approach for estimating muscle forces in an indeterminate biomechanical model. J Biomech 27: 1287-1289.

11. Cholewicki J, McGill SM, Norman RW (1995) Comparison of muscle forces and joint load from an optimization and EMG assisted lumbar spine model: towards development of a hybrid approach. J Biomech 28: 321-331.

12. Granata KP, Lee PE, Franklin TC (2005) Co-contraction recruitment and spinal load during isometric trunk flexion and extension. Clin Biomech (Bristol, Avon) 20: 1029-1037.

13. Pedotti A, Krishnan VV, Stark L (1978) Optimization of muscle-force sequencing in human locomotion. Mathematical Biosciences 38: 57-76.
14. Arnold AS, Salinas S, Asakawa DJ, Delp SL (2000) Accuracy of muscle moment arms estimated from MRI-based musculoskeletal models of the lower extremity. Computer Aided Surgery 5: 108-119.

15. Herzog W, Read LJ (1993) Lines of action and moment arms of the major force-carrying structures crossing the human knee joint. J Anat 182: 213-230.

16. Maganaris CN (2001) Force-length characteristics of in vivo human skeletal muscle. Acta Physiol Scand 172: 279-285.

17. Spoor CW, van Leeuwen JL, Meskers CG, Titulaer AF, Huson A (1990) Estimation of instantaneous moment arms of lower-leg muscles. J Biomech 23: $1247-1259$.

18. Reid SM, Lynn SK, Musselman RP, Costigan PA (2007) Knee biomechanics of alternate stair ambulation patterns. Med Sci Sports Exerc 39: 2005-2011.

19. Stokes M, Young A (1984) The contribution of reflex inhibition to arthrogenous muscle weakness. Clin Sci (Lond) 67: 7-14.

20. Kumar D, Karampinos DC, MacLeod TD, Lin W, Nardo L, et al. (2014) Quadriceps intramuscular fat fraction rather than muscle size is associated with knee osteoarthritis. Osteoarthritis Cartilage 22: 226-234.

21. McAlindon TE, Snow S, Cooper C, Dieppe PA (1992) Radiographic patterns of osteoarthritis of the knee joint in the community: the importance of the patellofemoral joint. Ann Rheum Dis 51: 844-849.

22. Shenoy R, Pastides PS, Nathwani D (2013) (iii) Biomechanics of the knee and TKR. Orthopaedics and Trauma 27: 364-371.

23. Hurwitz DE, Ryals AB, Case JP, Block JA, Andriacchi TP (2002) The knee adduction moment during gait in subjects with knee osteoarthritis is more closely correlated with static alignment than radiographic disease severity, toe out angle and pain. J Orthop Res 20:101-107.

24. Wada M, Imura S, Baba H, Shimada S (1996) Knee laxity in patients with osteoarthritis and rheumatoid arthritis. $\mathrm{Br} \mathrm{J}$ Rheumatol 35: 560-563. 